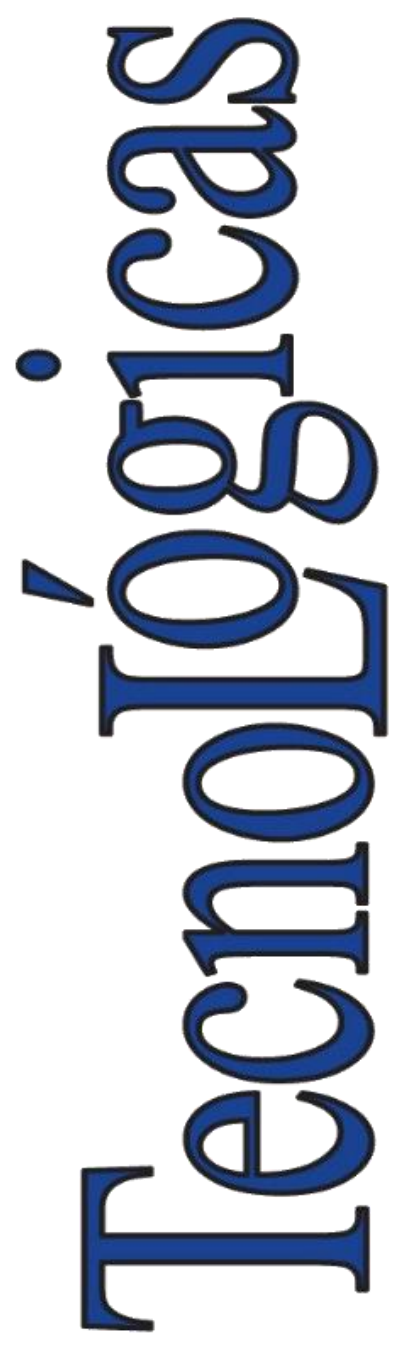

ISSN-p: 0123-7799 ISSN-e: $2256-5337$

Vol. 24, nro. 51, e1789, 2021

Recibido: 23 octubre 2020 Aceptado: 26 enero 2021

Disponible: 19 marzo 2021

CInstituto Tecnológico Metropolitano

Este trabajo está licenciado bajo una Licencia Internacional

Creative Commons Atribución (CC BY-NC-SA)

\section{Sedimentabilidad de partículas floculentas en aguas con alto contenido de color y baja turbiedad, coaguladas con $\mathrm{FeCl}_{3}+$ PAC versus PAC}

\section{Sedimentability of Flocculent Particles in Highly Colored Low Turbidity Water Coagulated with $\mathrm{FeCl}_{3}+\mathrm{PAC}$ Versus PAC}

Javier Fernández ${ }^{1}$; Susana Montenegro²; (iD) Cristina Ledezma ${ }^{3}$; (iD) Jeffrey Yanza*;

1 Universidad del Cauca, Popayán- Colombia, jefernandez@unicauca.edu.co

2 Universidad del Cauca, Popayán- Colombia, lsmontenegro@unicauca.edu.co

3 Universidad del Cauca, Popayán- Colombia, cledezma@unicauca.edu.co

${ }^{4}$ Universidad del Cauca, Popayán- Colombia, jeffrey@unicauca.edu.co

Cómo citar / How to cite

J. Fernández; S. Montenegro; C. Ledezma; J. Yanza, "Sedimentabilidad de partículas floculentas en aguas con alto contenido de color y baja turbiedad, coaguladas con $\mathrm{FeCl}_{3}+\mathrm{PAC}$ versus PAC", TecnoLógicas, vol. 24, nro. 51, e1789, 2021. https://doi.org/10.22430/22565337.1789 


\title{
Resumen
}

Las fuentes naturales con alto contenido de color y baja turbiedad, características de una fuente superficial de páramo, pueden limitar el proceso de una planta potabilizadora, ya que se genera una baja velocidad de sedimentación de los flóculos formados en el proceso de coagulación. El objetivo de este documento es presentar el resultado de la investigación realizada al comparar la sedimentación producida por la combinación de cloruro férrico $\left(\mathrm{FeCl}_{3}\right)$ e hidroxicloruro de aluminio (PAC), usados como coagulante primario y auxiliar de coagulación, respectivamente, versus PAC como coagulante primario. El estudio se desarrolló a escala de laboratorio utilizando pruebas de jarras y un agua natural con las características previamente mencionadas. Para cada opción de tratamiento se determinaron las curvas de sedimentación y se ajustaron a regresiones lineales, las cuales fueron comparadas estadísticamente. Asimismo, para cada curva, se estableció la eficiencia y la turbiedad teórica para tres velocidades de sedimentación: $1.3 \mathrm{~cm} / \mathrm{s}, 0.9 \mathrm{~cm} / \mathrm{s}$ y $0.6 \mathrm{~cm} / \mathrm{s}$. Los resultados indicaron que los dos procesos eran diferentes $(\mathrm{p}=0.000)$ y que el uso de $\mathrm{FeCl}_{3}+\mathrm{PAC}$ genera mayores velocidades de sedimentación de las partículas floculentas y menores turbiedades residuales teóricas, lo que permite mejorar la eficiencia del proceso de sedimentación. El estudio concluyó que el uso de $\mathrm{FeCl}_{3}$ en combinación con PAC representa una mejor opción técnica en comparación con el PAC como coagulante primario, ya que además de permitir la optimización del proceso de sedimentación, podría ayudar a aumentar la filtración y reducir el consumo de agua de lavado de filtros.

\section{Palabras Claves}

Baja turbiedad, sedimentabilidad de partículas, cloruro férrico, hidroxicloruro de aluminio.

\begin{abstract}
Highly colored low turbidity water, which is characteristic of high mountain sources, can limit the process of water treatment plants because it generates a low sedimentation rate of the flocs formed in the coagulation process. This article compares the sedimentation produced by the combination of ferric chloride $\left(\mathrm{FeCl}_{3}\right)$ and polyaluminium chloride (PAC) used as primary coagulant and coagulation auxiliary, respectively, versus PAC as primary coagulant. This study was conducted at laboratory level using jar tests and natural water with the aforementioned characteristics. The sedimentation curves of each treatment option were determined and adjusted to linear regressions, which were statistically compared. For each curve, efficiency and theoretical turbidity were determined using three sedimentation rates: $1.3 \mathrm{~cm} / \mathrm{s}, 0.9 \mathrm{~cm} / \mathrm{s}$, and $0.6 \mathrm{~cm} / \mathrm{s}$. The results indicate that the two processes were different $(\mathrm{p}=0.000)$, and the use of $\mathrm{FeCl}_{3}+\mathrm{PAC}$ generated higher sedimentation rates and lower theoretical residual turbidities, which improves the efficiency of the sedimentation process. In conclusion, the use of $\mathrm{FeCl}_{3}$ in combination with $\mathrm{PAC}$ represents a better technical option than PAC as primary coagulant because, in addition to optimizing the sedimentation process, it could help to increase the filtration run and reduce the consumption of water for filter washing.
\end{abstract}

\section{Keywords}

Low turbidity, sedimentability of particles, ferric chloride, polyaluminium chloride. 


\section{INTRODUCCIÓN}

Las aguas naturales con alto contenido de color y baja turbiedad presentan limitaciones para su tratamiento por coagulación química debido a las bajas tasas de sedimentación que presentan los flóculos generados por la baja concentración de partículas en suspensión y el alto contenido de materia orgánica natural (MON) que ocasiona la presencia de color real.

La MON es una mezcla compleja de partículas orgánicas de diferentes tamaños, pesos moleculares, estructuras, grupos funcionales e hidrofobicidad [1], pero compuesta principalmente de sustancias húmicas $(\mathrm{SH})$ : ácidos húmicos y fúlvicos formados a partir de procesos biológicos naturales de degradación de compuestos orgánicos, generalmente restos aromáticos, y de la interacción entre la materia vegetal y compuestos del suelo como la arcilla [2],[3]. La MON afecta directamente el agua potable, otorgándole color y sabor. Esta, si no es removida previamente, puede reaccionar con desinfectantes de uso común como el cloro y generar subproductos de desinfección (DBPs, por su sigla en inglés) que volverían potencialmente tóxicos, cancerígenos, mutagénicos o teratogénicos [4],[5],[6], tales como trihalometanos, ácidos acéticos halogenados, acetonitrilos halogenados, mutágeno X (MX), hidrato de cloral y clorofenoles, entre otros [7].

Adicionalmente, la MON puede ser alimento para el desarrollo de biopelículas en las tuberías que generaría un rebrote bacteriano en la red de distribución, disminuyendo la eficiencia de las operaciones de tratamiento del agua [8]. En las plantas de tratamiento de agua potable (PTAP), la eliminación del color es una tarea difícil que no asegura una eficiente remoción que perdure en el tiempo y es económicamente costosa [9],[10]. Esta situación se acentúa aún más si el agua presenta bajos valores de turbiedad y alcalinidad [11]. Una de las principales consecuencias técnicas de lo anterior es la baja sedimentabilidad de las partículas que, pese a ser desestabilizadas en el proceso de coagulación-floculación, generan pequeños flóculos $(<0.45 \mu \mathrm{m})$ con masas específicas bajas (30 KDa a $100 \mathrm{KDa}$ ) [12],[13], lo que limita su eliminación por sedimentación, lo cual a su vez se traduce en la disminución de la eficiencia del sistema y causa carreras de filtración más cortas [14],[15].

Latinoamérica, y especialmente Colombia, cuenta con circunstancias climáticas especiales, suelos volcánicos poco profundos y porosos con alto contenido de MON; particularmente su topografía, formada por la acción de los glaciales, genera una hidrología superficial como las fuentes de páramo [16]. Estas aguas poseen características fisicoquímicas propias (alto color, baja turbiedad, baja alcalinidad y ligeramente ácidas)[16] y se utilizan para abastecer a un número importante de comunidades urbanas y rurales, en las cuales se requiere optimizar el uso de tecnologías convencionales de tratamiento con coagulación química. Esto lleva a buscar alternativas que permitan la optimización de las operaciones unitarias de las PTAP, particularmente que mejoren la operatividad de los sedimentadores, para las cuales las tecnologías desarrolladas en Europa o Norte América, como por ejemplo la flotación, que mejora la remoción de las partículas floculentas en comparación con la sedimentación, no son aplicables completamente; sin embargo, genera mayores costos por consumo de energía por inyección de aire, lo cual en Colombia podría implicar mayores costos en la tarifa, especialmente en municipios pequeños y zona rural del país, además de la baja confiabilidad en la continuidad del suministro de energía en estas localidades.

En el año 2016 se realizó un estudio sobre la tratabilidad del agua del río Palacé, fuente de abastecimiento de una de las plantas de la ciudad de Popayán. La calidad del agua presentaba características propias de fuentes de páramo. Los valores promedio reportados de turbiedad y color del agua cruda fueron 7.34 UNT y 43.41 UPC, respectivamente, valores de $\mathrm{pH}$ en el rango de 6.88 a 7.33 . El estudio mostró que para tener eficiencias mayores al $85 \%$ en los sedimentadores, se requieren velocidades de sedimentación en los flóculos en el rango 
de $9.2 \mathrm{~m} / \mathrm{d}$ a $14.4 \mathrm{~m} / \mathrm{d}$, lo cual equivale a considerar cargas superficiales menores a $70 \mathrm{~m} 3 / \mathrm{m}^{2} / \mathrm{d}$ mediante la coagulación de $\mathrm{FeCl}_{3}+\mathrm{PAC}$; este último como ayudante de coagulación [17]. Estas cargas superficiales son más bajas que las recomendadas por la reglamentación colombiana para el diseño de los sedimentadores de alta tasa $\left(120 \mathrm{~m}^{3} / \mathrm{m}^{2} / \mathrm{d}\right.$ a $\left.185 \mathrm{~m}^{3} / \mathrm{m}^{2} / \mathrm{d}\right)$ [18], situación que pone en evidencia la necesidad de mejorar los procesos. Asimismo, trabajando con el agua de la misma PTAP, se comprobó que una combinación entre $\mathrm{FeCl}_{3}$, como coagulante primario, y policloruro de aluminio (PAC), usado como auxiliar de coagulación en pruebas de jarras, alcanzan al final del proceso rangos de color entre 1.0 UPC y 8.0 UPC y de turbiedad entre 0.65 UNT y 2.9 UNT, lo que representó eficiencias cercanas al $86.9 \%$ y $80.2 \%$, respectivamente [17].

Este hallazgo motivó la necesidad de realizar un estudio comparativo de la velocidad de sedimentación de las partículas coaguladas con $\mathrm{FeCl}_{3}+\mathrm{PAC}$ como coadyuvante de coagulación versus PAC como coagulante primario en agua natural, con alto contenido de color y baja turbiedad, en relación con la eficiencia de remoción de la turbiedad.

\section{Metodología}

El estudio se realizó en laboratorios utilizando el equipo de prueba de jarras y agua cruda del río Palacé, el cual sirve como fuente de abastecimiento a la PTAP que surte a la zona norte de la ciudad de Popayán. La PTAP tiene una capacidad para tratar $500 \mathrm{~L} / \mathrm{s}$; cuenta con las siguientes estructuras de tratamiento: mezcla rápida con vertedor frontal, floculador hidráulico, sedimentador de alta tasa con placas paralelas, filtros a tasa declinante y dosificación de cloro; utiliza PAC como coagulante primario. La calidad del agua cruda se caracteriza por presentar altos niveles de color y baja turbiedad [19], reportando para el periodo comprendido entre julio de 2012 y mayo de 2013, valores promedio de color aparente y turbiedad de 51.5 UPC y 17.9 UNT, respectivamente. Por otra parte, según los reportes diarios del año 2016, la planta de tratamiento Palacé reportó valores promedio de color de 43.4 UPC y 7.4 UNT de turbiedad [17].

Para evaluar la sedimentación del PAC, usado como coagulante primario, versus $\mathrm{FeCl}_{3}$ como coagulante y PAC como auxiliar de coagulación, se partió de la comprobación de los parámetros óptimos establecidos en estudios previos por [17], [19].

Para el estudio, la verificación y determinación de estos parámetros se realizó en un equipo de jarras EyQ Modelo F6-300-T (jarras cuadradas de 2 L de capacidad sin deflectores). Siguiendo la norma técnica colombiana NTC 3903 [20], con el fin de precisar la dosis se partió de una solución estándar del $1.00 \%$ p/v para el $\mathrm{FeCl}_{3}$ y una solución $0.34 \%$ p/v de PAC, suministrada por el proveedor de la planta. Para determinar el gradiente se utilizaron las curvas de correlación de velocidad de giro (RPM) vs gradiente de velocidad $\left(\mathrm{G}, \mathrm{s}^{-1}\right)$ para agua, generada por [21]. Los resultados se muestran en la Tabla 1.

La dosis de los coagulantes a utilizar en cada caso se determinó mediante una prueba de jarras, considerando las condiciones de calidad del agua cruda en el momento de realizar el ensayo. Para la dosis óptima del PAC como auxiliar de coagulación, se siguió la recomendación de [17], la cual se fijó $0.5 \mathrm{mg} / \mathrm{L}$. Para alcanzar las condiciones de $\mathrm{pH}$ óptimo de coagulación, fue necesario ajustar el $\mathrm{pH}$ del agua cruda a un valor de 8, utilizando para ello una solución de cal con una concentración $1 \mathrm{~N}$ de $\mathrm{Ca}(\mathrm{OH})_{2}$, dado que al dosificar el $\mathrm{FeCl}_{3}$ al agua se presenta una tendencia a disminuir el pH y limitar la eficiencia del proceso de coagulación. 
Tabla 1. Parámetros óptimos para prueba de jarras. Fuente: elaboración propia.

\begin{tabular}{cccc}
\hline Proceso & Parámetro & $\mathrm{FeCl}_{3}+\mathrm{PAC}$ & PAC \\
\hline \multirow{3}{*}{ Mezcla rápida } & Tiempo (s) & 20 & 10 \\
& Velocidad de giro (RPM) & 300 & 300 \\
& Gradiente $\left(\mathrm{s}^{-1}\right)$ & 360 & 360 \\
\cline { 2 - 4 } Mezcla lenta & Tiempo (min) & 20 & 30 \\
Sedimentación & Gradiente (RPM / s & $40 / 30$ & $40 / 30$ \\
\cline { 2 - 4 } & Tiempo $(\mathrm{min})$ & 25 & 25 \\
\hline
\end{tabular}

En total se realizaron cuatro curvas de sedimentabilidad para determinar cuál es la velocidad de sedimentación apropiada para el diseño de los sedimentadores. Para cada curva se consideraron tiempos de sedimentación de $10 \mathrm{~min}$ a $65 \mathrm{~min}$ (con intervalos de 5 min en cada jarra). Para cada punto de la curva, la velocidad de sedimentación se determinó dividiendo la altura de la toma de muestra en la jarra entre el correspondiente tiempo de sedimentación, mientras que la fracción restante de turbiedad se determinó como el cociente entre la turbiedad remanente en la jarra y la turbiedad inicial. Tanto al agua cruda como al efluente de cada jarra se le midió $\mathrm{pH}$, turbiedad y color, mediante el uso de un $\mathrm{pH}$ metro Orion 720, un turbidímetro Hach 2100Q y un colorímetro Hydrochem, respectivamente.

Dado que las curvas de sedimentación representan la eficiencia del proceso en función de la velocidad de sedimentación, se requiere saber si los dos tratamientos aplicados generan el mismo efecto o si presentan diferencias significativas, razón por la cual se realizaron dos tipos de comparaciones: regresión lineal entre las curvas de sedimentabilidad y pruebas t-pareadas para las turbiedades remanentes, estimadas con base en la eficiencia teórica de remoción, en tres velocidades de sedimentación específicas $(1.3 \mathrm{~cm} / \mathrm{min}, 0.9 \mathrm{~cm} / \mathrm{min}$ y $0.6 \mathrm{~cm} / \mathrm{min})$. Para ello se usó el paquete estadístico Statgraphics versión centurión XVI.

La eficiencia de remoción teórica se estimó mediante (1) y utilizando las curvas de sedimentabilidad desarrollada en cada prueba [15].

$$
E=1-C+\frac{1}{V s c} \int V s * d c
$$

\section{RESULTADOS}

La calidad del agua cruda para cada prueba, así como las dosis utilizadas se presentan en la Tabla 2. Las curvas de sedimentación encontradas en cada caso se presentan en las Figuras 1 a 4.

Tabla 2. Parámetros de calidad del agua cruda y dosis de coagulante. Fuente: elaboración propia.

\begin{tabular}{lcccc}
\multicolumn{1}{c}{ Parámetro } & \multicolumn{4}{c}{ Prueba } \\
& 1 & 2 & 3 & 4 \\
\hline pH crudo/ajustado & $7.5 / 8.9$ & $7.5 / 9.0$ & $7.3 / 8.9$ & $7.8 / 8.7$ \\
Alcalinidad (mg/L $\left.\mathrm{CaCO}_{3}\right)$ & 90.8 & 79.4 & 50.2 & 61.2 \\
Color (UPC) & 35.0 & 29.0 & 47.0 & 40.0 \\
Turbiedad (UNT) & 14.9 & 9.51 & 19.5 & 22.4 \\
Dosis FeCl $_{3}+\mathrm{PAC} *(\mathrm{mg} / \mathrm{L})$ & 39.0 & 48.0 & 42.0 & 42.0 \\
Dosis $\mathrm{PAC}(\mathrm{mg} / \mathrm{L})$ & 42.0 & 34.0 & 34.0 & 36.0 \\
\hline
\end{tabular}

*Dosis de PAC como coadyuvante $=0.5 \mathrm{mg} / \mathrm{L}$ en todas las pruebas. 


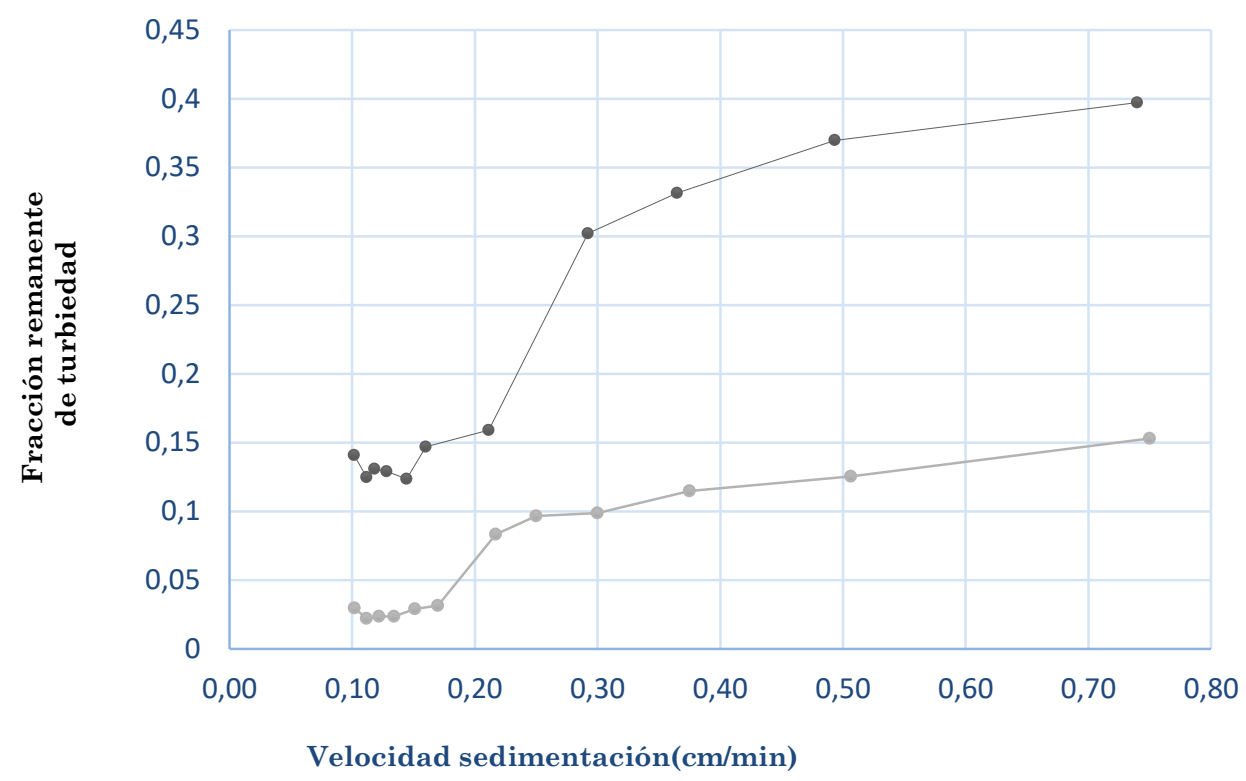

$\longrightarrow \mathrm{PAC} \longrightarrow \mathrm{FeCl} 3+\mathrm{PAC}$

Figura 1. Curvas de sedimentación prueba 1. Fuente: elaboración propia.

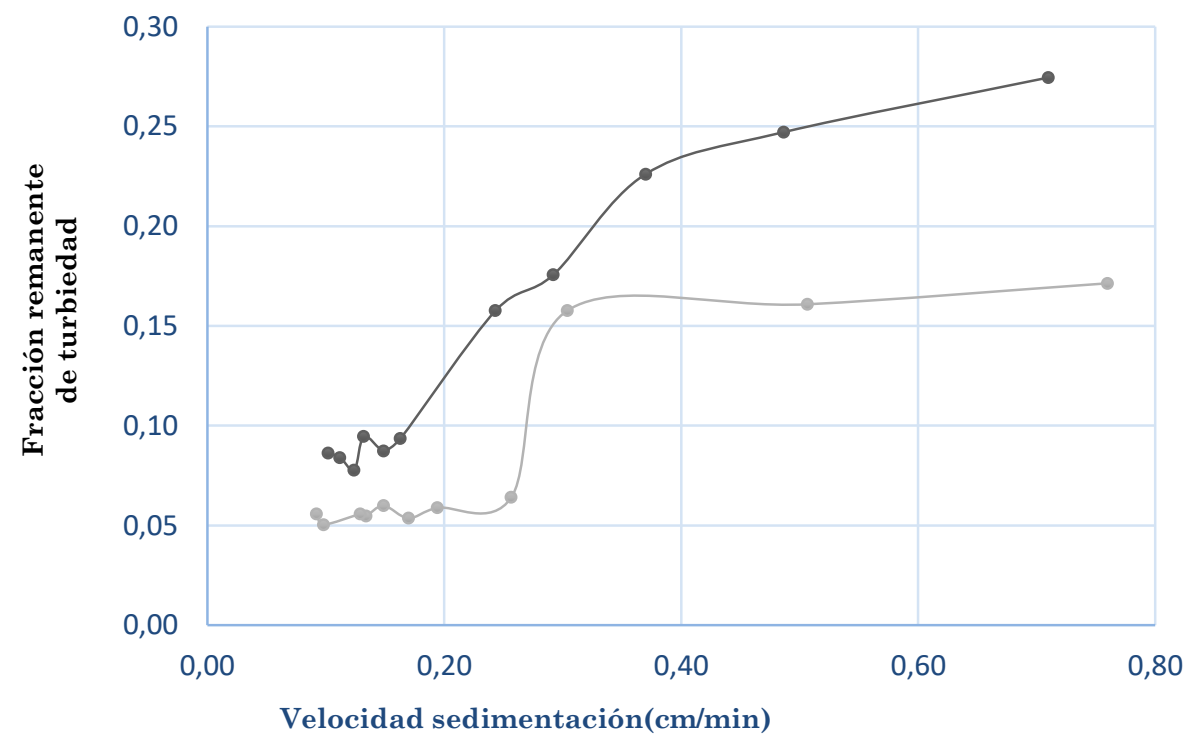

$\longrightarrow \mathrm{PAC} \longrightarrow \mathrm{FeCl} 3+\mathrm{PAC}$

Figura 2. Curvas de sedimentación prueba 2. Fuente: elaboración propia. 


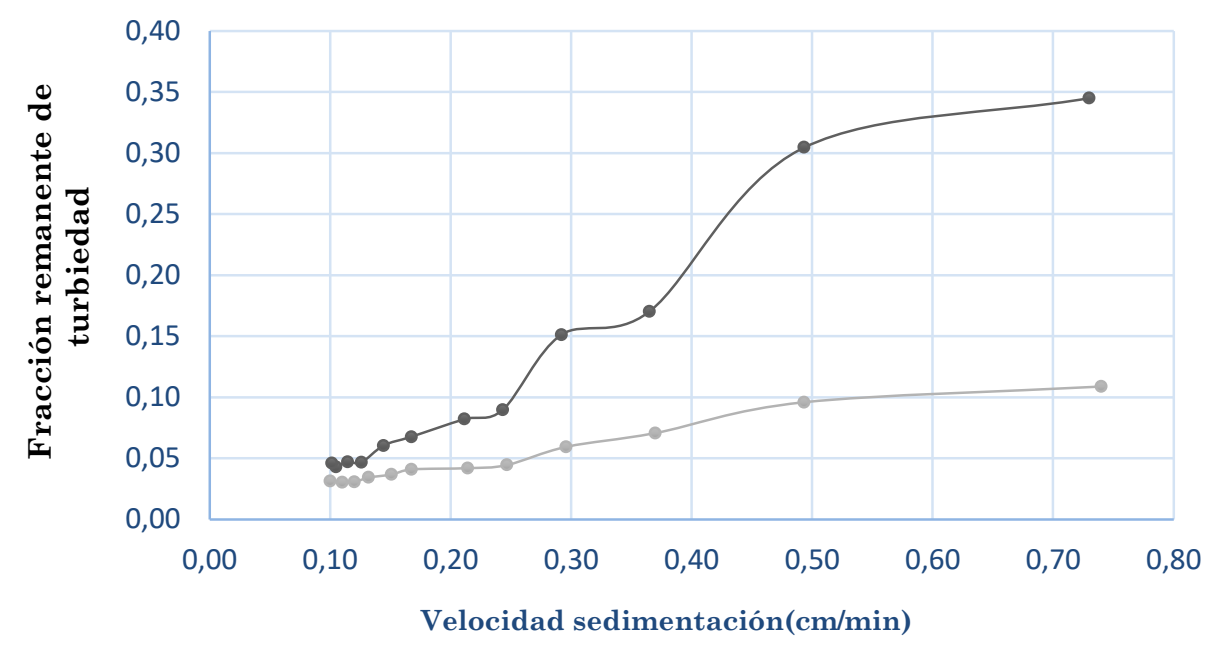

$\longrightarrow \mathrm{PAC} \longrightarrow \mathrm{FeCl} 3+\mathrm{PAC}$

Figura 3. Curvas de sedimentación prueba 3. Fuente: elaboración propia.

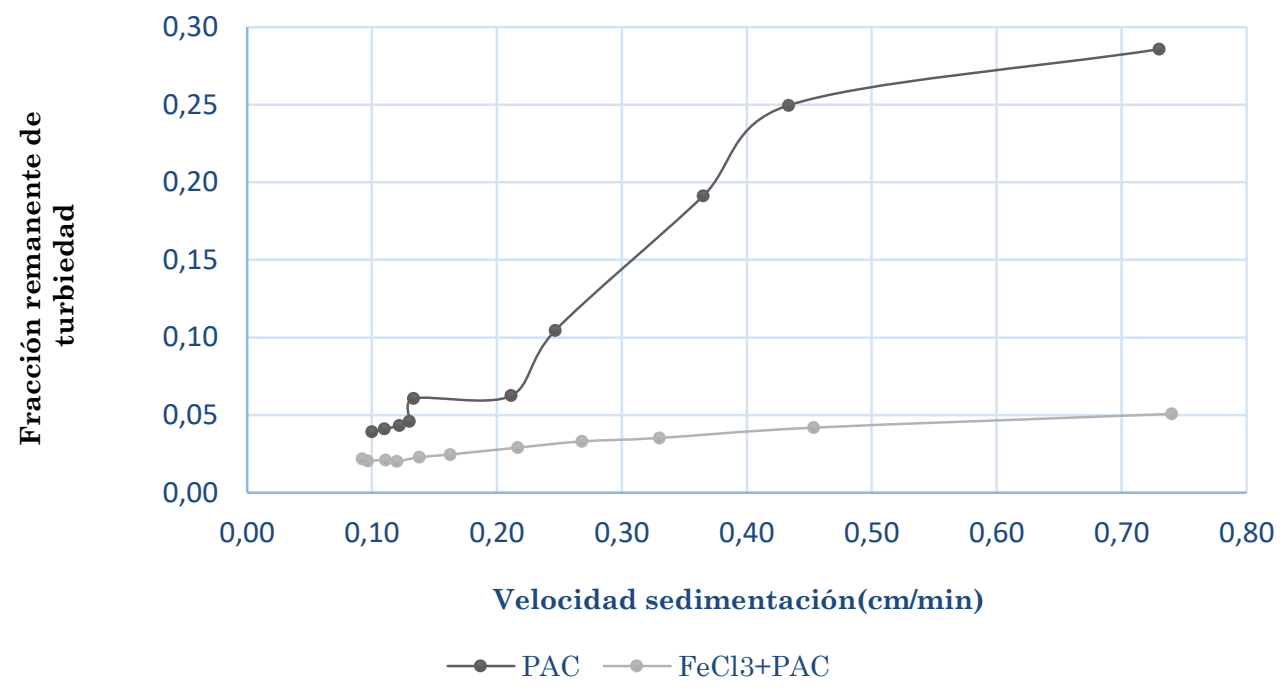

Figura 4. Curvas de sedimentación prueba 4. Fuente: elaboración propia.

Con el fin de realizar las comparaciones estadísticas, los datos de las curvas de sedimentación se ajustaron a un modelo logarítmico que describe de mejor manera el comportamiento de la curva, cuya ecuación (2) se expresa como:

$$
F r=a * \operatorname{Ln}(V s)+b
$$

Donde:

Fr: Fracción remanente

Vs: Velocidad de sedimentación ( $\mathrm{cm} / \mathrm{min})$

a: pendiente

b: Intercepto en la abscisa 
La Tabla 3 muestra los valores que se obtuvieron para cada uno de los tratamientos por cada ecuación ajustada. Los coeficientes de correlación de Pearson $\left(\mathrm{R}^{2}\right)$ estuvieron por encima del $90 \%$, lo cual explica la estrecha relación que existe entre la velocidad de sedimentación y la eficiencia de remoción de turbiedad.

Tabla 3. Valores de curvas de ajuste logarítmico por tratamiento. Fuente: elaboración propia.

\begin{tabular}{ccccc}
\hline Prueba & Tratamiento & $\mathrm{a}$ & $\mathrm{b}$ & $\mathrm{R}^{2}$ \\
\hline \multirow{2}{*}{1} & $\mathrm{PAC}$ & 0.160 & 0.461 & 0.922 \\
& $\mathrm{FeCl}_{3}+\mathrm{PAC}$ & 0.073 & 0.180 & 0.937 \\
\hline \multirow{2}{*}{2} & $\mathrm{PAC}$ & 0.112 & 0.319 & 0.964 \\
& $\mathrm{FeCl}_{3}+\mathrm{PAC}$ & 0.067 & 0.192 & 0.788 \\
\hline \multirow{2}{*}{3} & $\mathrm{PAC}$ & 0.153 & 0.360 & 0.891 \\
& $\mathrm{FeCl}_{3}+\mathrm{PACl}$ & 0.040 & 0.114 & 0.923 \\
\hline \multirow{2}{*}{4} & $\mathrm{PAC}$ & 0.134 & 0.326 & 0.924 \\
& $\mathrm{FeCl}_{3}+\mathrm{PAC}$ & 0.015 & 0.053 & 0.965 \\
\hline
\end{tabular}

En la Tabla 4 se muestran los resultados de la comparación estadística de regresión lineal entre las pendientes y los interceptos con los tipos de coagulante evaluados para las cuatro pruebas realizada. En particular, al comparar estadísticamente las regresiones lineales del PAC vs las del $\mathrm{FeCl}_{3}+\mathrm{PAC}$, se encontró que el valor-P, tanto para las pendientes como para los interceptos, es menor que 0.01 (ver Tabla 5), lo cual indica que existen diferencias significativas entre las pendientes y los interceptos de los tratamientos evaluados con un nivel de confianza del $99 \%$.

Tabla 4. Comparación estadística de regresión lineal entre las pendientes y los interceptos con los coagulantes evaluados. Fuente: elaboración propia.

\begin{tabular}{|c|c|c|c|c|c|c|}
\hline Prueba & Fuente & $\begin{array}{c}\text { Suma de } \\
\text { Cuadrados }\end{array}$ & $\mathrm{Gl}$ & $\begin{array}{c}\text { Cuadrado } \\
\text { medio }\end{array}$ & Razón-F & Valor-P \\
\hline \multirow{4}{*}{1} & Vel. Sed. & 0.041 & 1 & 0.041 & 589.9 & 0.000 \\
\hline & Interceptos & 0.182 & 1 & 0.182 & 2581.0 & 0.000 \\
\hline & Pendientes & 0.005 & 1 & 0.005 & 82.7 & 0.000 \\
\hline & Modelo & 0.229 & 3 & & & \\
\hline \multirow{4}{*}{2} & Vel. Sed. & 0.024 & 1 & 0.024 & 632.1 & 0.000 \\
\hline & Interceptos & 0.029 & 1 & 0.029 & 765.9 & 0.000 \\
\hline & Pendientes & 0.002 & 1 & 0.001 & 40.45 & 0.000 \\
\hline & Modelo & 0.052 & 3 & & & \\
\hline \multirow{4}{*}{3} & Vel. Sed. & 0.091 & 1 & 0.091 & 811.9 & 0.000 \\
\hline & Interceptos & 0.326 & 1 & 0.326 & 2899.4 & 0.000 \\
\hline & Pendientes & 0.028 & 1 & 0.028 & 251.35 & 0.000 \\
\hline & Modelo & 0.445 & 3 & & & \\
\hline \multirow{4}{*}{4} & Vel. Sed. & 0.075 & 1 & 0.075 & 640.31 & 0.000 \\
\hline & Interceptos & 0.623 & 1 & 0.623 & 5290.84 & 0.000 \\
\hline & Pendientes & 0.048 & 1 & 0.048 & 413.47 & 0.000 \\
\hline & Modelo & 0.747 & 3 & & & \\
\hline
\end{tabular}


Tabla 5. Turbiedad remanente por cada tratamiento según velocidad de sedimentación Fuente: elaboración propia.

\begin{tabular}{ccccccc}
\hline & \multicolumn{3}{c}{ Turbiedad PAC (UNT) } & \multicolumn{3}{c}{ Turbiedad $\mathrm{FeCl}_{3}+\mathrm{PAC}(\mathrm{UNT})$} \\
\hline Vs (cm/min) & 1.30 & 0.90 & 0.60 & 1.30 & 0.90 & 0.60 \\
Prueba 1 & 5.33 & 4.53 & 3.69 & 1.96 & 1.59 & 1.21 \\
Prueba 2 & 2.32 & 1.96 & 1.59 & 1.41 & 1.19 & 0.97 \\
Prueba 3 & 5.16 & 4.23 & 3.28 & 1.74 & 0.93 & 1.24 \\
Prueba 4 & 5.40 & 4.45 & 3.49 & 0.98 & 0.88 & 0.77 \\
\hline Media (UNT) & 4.55 & 3.79 & 3.01 & 1.52 & 1.15 & 1.05 \\
Desv. Est & 1.49 & 1.23 & 0.96 & 0.43 & 0.33 & 0.22 \\
\hline
\end{tabular}

Tal como lo muestra la Tabla 5, se evidencia que para el PAC los valores de turbiedad remanente se encontraron por encima de 3.0 UNT, mientras que para la combinación $\mathrm{FeCl}_{3}+\mathrm{PAC}$ no superaron 1.6 UNT. Considerando que estas turbiedades serán las de entrada a los filtros rápidos y que su valor genera una influencia tanto en la duración de carrera como en la turbiedad del agua filtrada [22],[23],[24], se debe analizar su impacto sobre estas dos variables. En general, los filtros se diseñan para alcanzar duraciones de carrera superiores a 24 h, reportándose valores en el rango de 24 h a 60 h [25]. En este sentido, la utilización de $\mathrm{FeCl}_{3}+\mathrm{PAC}$ comparado PAC, podría generar carreras de filtración más largas, lo cual disminuiría los costos de operación del sistema de tratamiento por los menores consumos de agua de lavado en los filtros.

Si bien el efecto más importante se presenta sobre la remoción de turbiedad, el color aparente también fue removido de manera significativa. Con velocidad de sedimentación máxima de $0.74 \mathrm{~cm} / \mathrm{min}$, se alcanzaron valores promedio al final del proceso de sedimentación de 18.8 UPC y 14.0 UPC, equivalente a una remoción de 54.0 \% y $59.2 \%$ para PAC y $\mathrm{FeCl}_{3}+\mathrm{PAC}$, respectivamente. En este sentido, los dos tratamientos evaluados cumplirían la normatividad colombiana sobre color (máximo 15 UPC) [26], dado que en el proceso de tratamiento aún faltaría considerar la remoción generada por la filtración.

Por otra parte, los ministerios de Salud y Protección Social, Ambiente y Desarrollo Sostenible y Vivienda, Ciudad y Territorio de Colombia [26], indican que se debe tener turbiedades por debajo de 2 UNT en el agua suministrada para consumo humano, que podría ser garantizado en mayor medida con la utilización del $\mathrm{FeCl}_{3}+\mathrm{PAC}$ que con el PAC; incluso se podrían alcanzar turbiedades por debajo de 0,5 UNT, tal como lo recomienda la Organización Mundial de la Salud [27] para asegurar una efectiva desinfección y reducir el riesgo microbiológico por Cryptosporidium si se alcanza un percentil 95 de 0.15 UNT; igualmente la EPA [28] refiere de un máximo de 0.3 UNT para la desinfección efectiva. Mantener baja la turbiedad del agua filtrada, además de reducir el riesgo microbiológico, permite el uso de dosis más bajas de desinfectante y reduce la formación de subproductos de desinfección [29].

\section{CONCLUSIONES}

En aguas con alto color y baja turbiedad, como la que se presenta en el río Palacé, el estudio concluyó que el uso de FeCl3 como coagulante primario y PAC como ayudante de coagulación representan una mejor opción técnica en comparación con el PAC como coagulante primario, ya que genera flóculos con mayores velocidades de sedimentación que mejoran la eficiencia de remoción en turbiedad y permiten la optimización de la sedimentación. Respecto a la remoción de color, los dos tratamientos evaluados podrían cumplir la normatividad colombiana. 
Adicionalmente, esta opción podría generar menores costos en la dosificación de productos químicos y ahorro en el agua de lavado de los filtros. En este sentido, los autores recomiendan la realización de un estudio en planta escala real que permita la validación de los aspectos antes mencionados.

\section{AGRADECIMIENTOS}

A la empresa de Acueducto y Alcantarillado de Popayán S.A. E.S.P. y al ingeniero Mauricio Ramírez y operarios de la PTAP de Palacé por facilitar los equipos de medición de laboratorio y los espacios en general de la planta de tratamiento para el desarrollo del presente estudio. Este estudio contó con apoyo económico de la Universidad del Cauca, quien asumió el costo de los docentes participantes en la investigación.

\section{CONFLICTO DE INTERÉS}

Los autores declaran que no tienen intereses económicos en competencia o relaciones personales que pudieran haber influido en el trabajo reportado en este documento.

\section{CONTRIBUCIÓN DE AUTORES}

Javier Fernández: definición de la metodología, coordinación del equipo de trabajo, manejo estadístico de los datos, apoyo en la redacción del documento, revisión y aprobación final de la escritura y correcciones técnicas del manuscrito.

Susana Montenegro: desarrollo de la metodología en cuanto a pruebas experimentales y adquisición de los datos, apoyo en la escritura y preparación del documento y correcciones del manuscrito.

Cristina Ledezma: desarrollo de la metodología en cuanto a pruebas experimentales y adquisición de los datos, apoyo en la escritura y preparación del documento y correcciones del manuscrito.

Jeffrey Yanza: desarrollo de la metodología en cuanto a pruebas experimentales y adquisición de los datos, procesamiento y manejo estadístico de los datos y apoyo en la escritura del manuscrito y preparación del documento.

\section{REFERENCIAS}

[1] H. C. Lin; G. S. Wang, "Effects of UV/H2O2 on NOM fractionation and corresponding DBPs formation," Desalination, vol. 270, no. 1-3, pp. 221-226, Apr. 2011. https://doi.org/10.1016/j.desal.2010.11.049

[2] G. A. Edwards; A. Amirtharajah, "Removing Color Caused By Humic Acids.," J. / Am. Water Work. Assoc., vol. 77, no. 3, pp. 50-57, Mar. 1985. https://doi.org/10.1002/j.1551-8833.1985.tb05508.x

[3] P. Finkbeiner; G. Moore; R. Pereira; B. Jefferson; P. Jarvis, "The combined influence of hydrophobicity, charge and molecular weight on natural organic matter removal by ion exchange and coagulation," Chemosphere, vol. 238, pp. 124633, Jan. 2020. https://doi.org/10.1016/j.chemosphere.2019.124633

[4] E. P. Tangerino; L. Di Bernardo, "Remoção de substâncias húmicas por meio da oxidação com ozônio e peróxido de hidrogênio e FiME," Eng. Sanit. e Ambient., vol. 10, no. 4, pp. 290-298, 2005. https://doi.org/10.1590/S1413-41522005000400005

[5] J. J. Rook, "Chlorination reactions of fulvic acids in natural waters," Environ. Sci. Technol., vol. 11, no. 5, pp. 478-482, May 1977. https://doi.org/10.1021/es60128a014 
[6] H. Selcuk; S. Meric; A. Nikolaou; M. Bekbolet, "A comparative study on the control of disinfection byproducts (DBPs) and toxicity in drinking water," Desalin. Water Treat. - desalin water treat, vol. 26, pp. 165-171, Aug. 2012. https://doi.org/10.5004/dwt.2011.2126

[7] M. T. Olmedo Sanchez, "Subproductos de la desinfección del agua por el empleo de compuestos de cloro. Efectos sobre la salud," Hig. Sanid. Ambient., vol. 342, pp. 335-342, 2008. URL

[8] J. Yli-Kuivila; I. T. Miettinen; R. Laukkanen, "Potential of ferric and polyaluminium coagulants for nanofiltration pretreatment," in Chemical Water and Wastewater Treatment, vol. 6, H. H. Hahn; E. Hoffmann; H. Odegaard, eds. Springer, 2000, pp. 181-190. https://doi.org/10.1007/978-3-642-59791-6 17

[9] W. L. Ang; A. W. Mohammad; A. Teow; A. Benamor; N. Hilal, "Hybrid chitosanFeCl3 Coagulationmembrane processes Performance evaluation and membrane fouling study in removing natural organic matter," Sep. Purif. Technol., vol. 152, pp. 23-31, Sep. 2015. https://doi.org/10.1016/j.seppur.2015.07.053

[10] M. Kumari; S. K. Gupta, "A novel process of adsorption cum enhanced coagulation-flocculation spiked with magnetic nanoadsorbents for the removal of aromatic and hydrophobic fraction of natural organic matter along with turbidity from drinking water," J. Clean. Prod., vol. 244, p. 118899, Jan. 2020. https://doi.org/10.1016/j.jclepro.2019.118899

[11] F. Worrall; T. Burt, "Predicting the future DOC flux from upland peat catchments," J. Hydrol., vol. 300 , no. 1-4, pp. 126-139, Jan. 2005. https://doi.org/10.1016/j.jhydrol.2004.06.007

[12] M. De Julio; T. S. De Julio; L. Di Bernardo, "Influence of the apparent molecular size of humic substances on the efficiency of coagulation using Fenton's reagent," An. Acad. Bras. Cienc., vol. 85, no. 2, pp. 833-847, Apr. 2013. https://doi.org/10.1590/S0001-37652013005000030

[13] L. Fusheng; Y. Akira; A. Yuka, "Characterization of micro-flocs of NOM coagulated by PACI, alum and polysilicate-iron in terms of molecular weight and floc size," Water Sci. Technol., vol. 57, no. 1, pp. 83-90, Jan. 2008. https://doi.org/10.2166/wst.2008.775

[14] L. Di Bernardo; C. G. da N. Mendes; A. F. Guimaraes, "Coagulaçao-floculaçao de águas com turbidez ou cor elevada-parte I e 2," Revista DAE, vol. 47, no. 150. pp. 227-231, 1987. URL

[15] J. Arboleda, Teoría y práctica de la purificación del agua. Bogotá, 1992. URL

[16] W. Buytaert; R. Célleri; B. De Biévre; F. Cisneros, "Hidrología del páramo andino: propiedades, importancia y vulnerabilidad,” pp. 1-26. URL

[17] J. Yanza-López; R. Rivera-Hernández; L. Gómez-Torres; C. Zafra-Mejía, "Evaluación de FeCl3 y PAC para la potabilización de agua con alto contenido de color y baja turbiedad," TecnoLógicas, vol. 22, no. 45, pp. 921, 2019. https://doi.org/10.22430/22565337.1085

[18] Ministerio de Vivienda, Ciudad y Territorio de Colombia, Resolución 0330 de 2017. 2017, p. 77.

[19] A. Muñoz; V. Valencia, "Estudio de los parámetros óptimos de tratabilidad para la fuente de abastecimiento de la planta Palacé, en el municipio de Popayán, departamento del Cauca," Universidad del Cauca, 2013.

[20] ICONTEC, "Procedimiento para el ensayo de coagulación-floculación en un recipiente con agua o método de jarras" Norma Tecnica Colombiana NTC 3903:2010, Apr. 2010. URL

[21] R. Lai; H. Hudson; J. Singley, "Velocity Gradient Calibration of Jar-Test Equipment." Journal (American Water Works Association), vol. 67, no. 10, pp. 553-557, 1975. URL

[22] H. Mahanna; M. Fouad; K. Radwan; H. Elgamal, "Predicting of Effluent Turbidity from Deep Bed Sand Filters Used in Water Treatment," Int. J. Sci. Eng. Res., vol. 6, no. 9, pp. 621-626, Sep. 2015. https://doi.org/10.14299/ijser.2015.09.006

[23] A. Upton; B. Jefferson; G. Moore; P. Jarvis, "Rapid gravity filtration operational performance assessment and diagnosis for preventative maintenance from on-line data," Chem. Eng. J., vol. 313, pp. 250-260, Apr. 2017. https://doi.org/10.1016/j.cej.2016.12.047

[24] H. Mahanna; K. Radwan; M. Fouad; H. Elgamal, "Effect of Operational Conditions on Performance of Deep sand Filter in Turbidity Removal," Trends Tech. Sci. Res., vol. 2, no. 5, pp. 1-7, Aug. 2018. URL

[25] D. Ratnayaka; M. Brandt; M. Johnson, Twort’s Water Supply. Elsevier, Burlington, USA, 2009.

[26] Ministerio de la protección social y Ministerio de ambiente, vivienda y desarrollo territorial, Resolución 2115, Junio de 2007. URL

[27] World Health Organization, Guidelines for Drinking-Water Quality, Second Edi. Hong Kong: WHO, 2002. URL

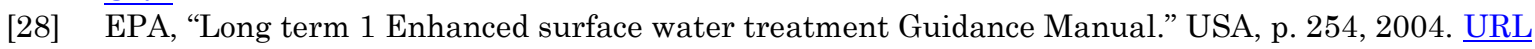

[29] P. Hulck; M. Elmelko, Filter operation effects on pathogen passage. Washington: AWWA Research Foundation, 2001. 